\title{
Hermeneutik als rationale Methodenlehre der Interpretation
}

\section{Mantzavinos}

in: Zeitschrift für philosophische Forschung, (im Erscheinen 2019)

\begin{abstract}
Ziel dieses Aufsatzes ist, $\mathrm{zu}$ zeigen, dass intersubjektive Verständlichkeit, Überprüfbarkeit mithilfe von Evidenz, Austausch von Argumenten und Objektivität im Falle der Textinterpretation möglich sind. Insofern man bereit ist, in der Anwendung solcher Maßstäbe die Wissenschaft als rationales Unternehmen anzuerkennen, wäre auch die Interpretation als ein rationales Unternehmen anzuerkennen und die Hermeneutik als rationale Methodenlehre der Interpretation zu bezeichnen.
\end{abstract}

The aim of this paper is to show that intersubjective intelligibility, testability with the use of evidence, rational argumentation and objectivity are possible in the case of text interpretation. As far as one is willing to accept that the application of such standards make up science as a rational enterprise, one should also accept text interpretation as a rational enterprise and should be willing to qualify hermeneutics as a rational methodology of interpretation 


\section{Einleitung}

Hermeneutik als die Methodologie der Interpretation befasst sich mit Problemen, welche bei der Behandlung von sinnhaften menschlichen Handlungen und den Ergebnissen solcher Handlungen, vor allem Texten, Bildern und anderen symbolischen Systemen, auftauchen. Als methodologische Disziplin bietet sie einen Werkzeugkasten, um die effiziente Behandlung der Probleme der Interpretation von menschlichen Handlungen, Texten und anderem sinnhaften Material zu erleichtern. Die Hermeneutik baut auf einer langen Tradition auf, da die Probleme mit denen sie sich auseinandersetzt, sehr verbreitet im menschlichen Leben sind und eine effektive Behandlung benötigen: die Interpretation ist eine allgegenwärtige Aktivität, welche sich entfaltet, wann immer Menschen versuchen, diejenigen interpretanda zu erfassen, die sie als relevant empfinden. Während ihrer langen Geschichte ist es nur natürlich, dass sowohl die Probleme als auch die Werkzeuge, die zu ihrer Lösung entwickelt worden sind, sich im Zeitverlaufe erheblich verändert haben - nebst der Hermeneutik als Disziplin. Ich werde mich in meinem heutigen Vortrag, auf die wichtigsten Problembereiche fokussieren und einige Vorschläge präsentieren, sie effektiv zu bewältigen.

Die Bandbreite der modernen Diskussionen über Interpretation ist sehr groß. Oft beginnt man mit der Frage, ob menschliche Handlungen als Naturphänomene betrachtet werden sollten oder nicht und wie man sie behandeln sollt. Naturalisten seit Mill (1873/1974, Book VI) vertreten die These, dass menschliche Handlungen im Kontinuum mit anderen Phänomenen in der Natur betrachtet werden sollten und dass man sie entsprechend erforschen soll. Interpretationsprobleme treten kaum zutage, wenn man eine solche Betrachtungsweise übernimmt. Im Gegensatz hierzu haben Interpretivisten wie Dilthey (1883/1990; 1924/1990; 1927/1992) vehement argumentiert, dass menschliche Handlungen nicht als Naturphänomene betrachtet werden können, da ihre Sinnhaftigkeit sie kategorial unterscheidet. Bloße Ereignisse, wie reine physiologische Reaktionen und Körperbewegungen, konstituieren keine menschlichen Handlungen - darüber herrscht

Konsens. Die Meinungsverschiedenheit bezieht sich auf die Frage, ob es für eine Handlung konstitutiv ist, dass sie sinnhaft ist, oder eher nicht (Mantzavinos, 2012). Wenn 
man die interpretivistische Position übernimmt, dann tauchen Interpretationsfragen zwangsläufig im Bereich des Mentalen auf. Menschliche Handlungen sind sinnhaft und die Ergebnisse von Handlungen konstituieren sinnhaftes Material, dass interpretationsbedürftig ist.

Es ist wichtig, sorgsam zwischen zwei Analyseebenen zu unterscheiden, der ontologischen und der epistemologischen. Heidegger hat eine hermeneutische Phänomenologie als eine Hermeneutik der Faktizität (1923/1995) vorgeschlagen, welche die traditionelle Ontologie ersetzen sollte: das Kernstück war die "existenziale Analyse des Daseins", d.h. der menschlichen Existenz (1927). Die Frage nach dem Sinn des Seins sollte mittels der Angabe sogenannter "Existenzialien" des Daseins beantwortet werden. Was heißt das? Es heißt, dass der Mensch bzw. die "Existenz" mit Hilfe von sogenannten "Existenzialien" analysiert wird, statt mit Hilfe von Kategorien; der Mensch ist also nicht als Fall einer Gattung von Dingen anzusehen, die neben anderen Dingen vorkommen, sondern genießt einen Sonderstatus, den es im Hinblick auf die Existenzidee zu analysieren gilt ${ }^{1}$. Hermeneutik ist demnach das Auslegen bzw. die Interpretation der Faktizität, d.h. des Menschen². Gadamer (1960/1990; 1986/1993; 2000) hat teilweise diese Betrachtungsweise über Ontologie übernommen, sodass sich die sogenannte philosophische Hermeneutik als philosophisches Programm - aufbauend hauptsächlich auf den Werken dieser beiden Protagonisten - entwickelt hat (Malpas und Gander 2014). Obwohl epistemologische Studien in der Hermeneutik sich gegenüber diesen oder anderen Thesen ontologischer Natur verpflichten können, brauchen sie dies nicht zu tun. Epistemologische Ansätze, seien sie deskriptiv oder normativ, können mit Problemen der Interpretation anfangen und Lösungen zu diesen Problemen vorschlagen und zwar

\footnotetext{
${ }^{1}$ Vgl. Für eine ausführliche Diskussion vgl. Mantzavinos (2006, S. 24ff.)

2 Vgl. Heidegger (1923/1995, S. 7): "Faktizität ist die Bezeichnung für den Seinscharakter 'unseres', 'eigenen' Daseins. Genauer bedeutet der Ausdruck: jeweilig dieses Dasein (Phänomen der 'Jeweiligkeit'; vgl. Verweilen, Nichtweglaufen, Da-bei, Da-sein), sofern es seinsmäßig in seinem Seinscharakter 'da' ist. Seinsmäßig dasein besagt: nicht und nie primär als Gegenstand der Anschauung und anschaulicher Bestimmung, der bloßen Kenntnisnahme von ihm, sondern Dasein ist ihm selbst da in Wie seines eigensten Daseins." Und weiter auf S. 15: "[D]ie Beziehung zwischen Hermeneutik und Faktizität ist dabei nicht die von Gegenstandserfassung und erfasstem Gegenstand, dem jene sich lediglich anzumessen hätte, sondern das Auslegen selbst ist ein mögliches ausgezeichnetes Wie des Daseinscharakters der Faktizität".
} 
unabhängig von der ontologischen Konstitution und Struktur, welche dem jeweiligen Problembereich zugrunde liegt.

\section{Verstehen vs. Erklären}

Auch wenn die Unterscheidung zwischen der ontologischen und der epistemologischen Analyseebene zum großen Teil anerkannt ist, ist es immer eine Streitfrage geblieben, ob es in der Tat fruchtbar ist, die Konstitution und Struktur des Materials mit welchem man konfrontiert ist, wenn man Interpretationsaktivitäten unternimmt, ganz außer Acht zu lassen. Die alte Kontroverse "Verstehen vs. Erklären" bezieht sich zum großen Teil auf diese Frage: ob es eine spezielle Methode zur Erfassung sinnhaften Materials gibt, die in den Geisteswissenschaften angewandt werden sollte - nämlich Verstehen - oder die allgemeine Methode, die in den Naturwissenschaften angewandt wird, auch in den Sozial- und Geisteswissenschaften einsetzbar ist - nämlich Erklären. Methodologische Dualisten wie Dilthey sind bekannt dafür, vehement für die Autonomie der Sozial- und Geisteswissenschaften zu plädieren, welche die Methode des Verstehens anzuwenden haben. Die Neo-Kantianer Wilhelm Windelband (1894) und Heinrich Rickert (1929) haben sich auf den Prozess der Begriffsbildung in den unterschiedlichen Gruppen von Wissenschaften fokussiert, den Kulturwissenschaften und den Naturwissenschaften. Für Windelband (1894) sind die Kulturwissenschaften vom idiographischen Interesse geprägt, sie haben mit dem Einmaligen, Individuellen und Besonderen zu tun, wobei die Naturwissenschaften nomothetisch verfahren, d.h. mit dem Auffinden allgemeiner Gesetze befasst sind.

Im Gegensatz zu dieser dualistischen Auffassung, lehnen methodologische Monisten wie Mill die Dichotomie ab und plädieren für eine einheitliche Methode, anwendbar in allen Wissenschaften - überzeugt, wie er, dass die Entdeckung und Etablierung gesetzesartiger Hypothesen auch in den Sozial- und Geisteswissenschaften möglich ist. Den Kern der Kontroverse (Ricoeur 1981; L. Anderson 2003) macht die Frage über die Akzeptanz 
dessen aus, was man das "Methode-Gegenstand-Argument" nennen kann ${ }^{3}$, d.h. der These, dass die wissenschaftliche Methode sich dem Gegenstand der Forschung anpassen muss. Wenn der Gegenstand der wissenschaftlichen Analyse, ontologisch gesehen, gewisse Eigenschaften aufweist, dann muss eine wissenschaftliche Methode in Anspruch genommen werden, die diesen Eigenschaften gemäß ist. Dieses Argument postuliert demnach den Primat des $\mathrm{zu}$ erforschenden Gegenstandes vor der Art seiner Erkenntniserfassung. Je nachdem wie man zur Überzeugungskraft dieses Arguments steht, übernimmt man entweder Verstehen oder Erklären, obwohl es natürlich auch sehr raffinierte Versuche gegeben hat, beides irgendwie unter einen Hut zu bringen - man braucht nur an Max Webers (1922/1985) "verstehendes Erklären" zu denken.

\section{Textinterpretation}

Es scheint prima facie plausibel zu postulieren, dass nichts Weiteres für das Verstehen eines Textes nötig ist, als das Verstehen der Sätze, die ihn bilden; und das nichts Weiteres für das Verstehen eines Satzes nötig ist, als das Verstehen der Wörter, die ihn bilden. Diese verbreitete Auffassung basiert auf dem Glauben der Gültigkeit des Prinzips der Kompositionalität des Sinnes: Der Sinn eines komplexen sprachlichen Ausdruckes ist vollständig von der Struktur und dem Sinn seiner Bestandteile determiniert (Szabo, 2013). Gottlob Frege hat bereits in der Sektion 60 seiner Grundlagen der Arithmetik (1884) deklariert, dass nur Sätze als Ganze Sinn haben. Dieses unterschiedliche, aber verwandte Prinzip zu dem Prinzip der Kompositionalität wird oft das Prinzip des Kontextes genannt. Er schreibt: "Es genügt, wenn der Satz als Ganzes einen Sinn hat; dadurch erhalten auch seine Theile ihren Inhalt".

Es gibt einen Konsens bei vielen Bedeutungstheorien, dass der semantische Gehalt eines Satzes eine Funktion des semantischen Gehaltes seiner Bestandteile ist - und insofern das Prinzip der Kompositionalität anwendbar ist. Der Versuchung ein analoges Prinzip für

\footnotetext{
${ }^{3}$ Vgl. Mantzavinos (2006, S. 13ff.).
} 
den Fall von Texten anzunehmen, sollte man jedoch widerstehen: der semantische Gehalt eines Textes ist keine Funktion des semantischen Gehaltes seiner Bestandteile und seiner Struktur. Während ein Satz einen Gedanken ausdrücken kann, der somit ein plausibles mentales Korrelat ausmacht, drückt ein Text eine Gedankensequenz aus, die nicht direkt erfassbar ist: den Sinn eines Satzes kann man erfassen, sich einprägen und verarbeiten; der Sinn eines Textes als Ganzes auf der Makro-Ebene fordert für sein Verständnis einen viel komplexeren kognitiven Prozess (Scholz, 2012).

Die Anerkennung der Komplexität des Textverstehens als eines Prozesses ist der erste Schritt, um nach Modellen Ausschau zu halten, welche mit dieser Komplexität erfolgreich umgehen können. Solche Modelle werden im Rahmen der kognitiven Psychologie diskutiert. Ein prominentes Beispiel eines solchen Modells ist von Kintsch und van Deijk (1978) vorgeschlagen worden und fokussiert sich auf den Informationsbearbeitungsprozess, der stattfindet, nachdem die Prozesse der syntaktischen und semantischen Analyse bereits vorgenommen worden sind, um den Text in eine Menge von Propositionen zu zergliedern. Das Modell konzentriert sich auf die weitere Verarbeitung des Textes, nachdem die Ausgangsmenge der Propositionen bereits identifiziert wurde. Sie gehen davon aus, dass es eine Kapazitätsbeschränkung bezüglich der Anzahl an Propositionen gibt, die man aktiv im Arbeitsgedächtnis behalten kann. Das hat zur Konsequenz, dass nur diejenigen Propositionen aktiv gehalten werden, die gemäß dem Kriterium der zeitlichen Nähe und der Wichtigkeit der Information für die Sinnermittlung des ganzen Textes relevant sind. Gemäß der sog. Leading-edge-Strategie halten die Probanden diejenige Proposition aktiv, die gerade zuvor verarbeitet wurde, sowie weitere Propositionen, die in der hierarchischen Darstellung eines Textes dieser Proposition übergeordnet sind, weil sie davon ausgehen, dass es hierarchische Verbindungen zwischen den Propositionen eines Textes gibt. Wichtig ist, dass parallel dazu ein Elaborationsprozess abläuft. Bei diesem finden einerseits Überbrückungsinferenzen statt, bei denen zu den jeweils vorhandenen Propositionen schlussfolgernd erschlossene Verknüpfungen hinzugefügt werden, um ansonsten nicht zustande gekommene Verknüpfungen herzustellen. Andererseits werden sog. Makropropositionen gebildet, die eine Zusammenfassung des Wesentlichen im Text 
beinhalten. Während dieses Elaborationsprozesses wird der Text seitens des Interpreten aktiv gedeutet und somit "verstanden". ${ }^{4}$

Solche Modelle des Textverstehens sind empirisch getestet und stellen einen großen Schritt vorwärts in Richtung einer Darstellung vom Textverstehen, das auf solider empirischer Evidenz basiert, dar. Eine übliche philosophische Kritik hinterfragt jedoch die Möglichkeit, überprüfbare Modelle von Textinterpretation zu konzipieren ohne vorher die normativen Voraussetzungen anzuerkennen, die jeglicher Interpretationspraxis zugrunde liegen. Es gibt zwei Argumentationslinien, die in diesem Zusammenhang einflussreich gewesen sind. Die erste wurde emphatisch innerhalb der AngloAmerikanischen philosophischen Diskussion der zweiten Hälfte des 20. Jahrhunderts propagiert und zwar im Hinblick auf das, was als "radikale Interpretation" bekannt geworden ist. In einer imaginären Situation wird ein Interpret mit dem sprachlichen Verhalten einer Person konfrontiert, in einer komplett fremden Kultur, ohne jegliche Kenntnis über ihre Überzeugungen, Wünsche und die Bedeutungen dessen, was diese Person aussagt. Das Problem besteht darin, die Überzeugungen, Wünsche und Bedeutungen dieser Person in Erfahrung zu bringen und zwar indem man ganz von vorne anfängt, mit anderen Worten indem man die Person als ein physisches System ohne jegliche Hilfe bei der Interpretation betrachtet (Lewis, 1983, 108). Im Kontext dieses weitgehend künstlichen Problems wird behauptet, dass man verpflichtet sei, ein allgemeines Interpretationsprinzip normativer Natur zu übernehmen, dass vermeintlich

\footnotetext{
${ }^{4}$ Vgl. auch die zusammenfassende Beschreibung des Modells, das von Kintsch (1998, S. 119) das "Konstruktion-Integration Modell" genannt wird: "Ein Prozess-Modell des Textverständnisses versucht einen Schritt-für-Schritt Prozess zu beschreiben, durch den geschriebene oder gesprochene Sprache in eine mentale Repräsentation im Geiste des Lesers oder des Hörers transformiert wird. Das Konstruktion-Integration - (KI-) Modell nimmt an, dass dieser Prozess zwei Phasen beinhaltet: eine Konstruktionsphase, in der ein approximatives, aber inkohärentes mentales Modell aus dem Textinput und den Zielen und Wissen des Verstehenden lokal konstruiert wird, und eine Integrationsphase, die im Wesentlichen ein EinschränkungsErfüllungs-Prozess ist, der unpassende lokale Konstruktionen ablehnt, zugunsten von denen, die zusammen ein kohärentes Ganzes ergeben. Die Konstruktionsregeln in diesem Modell können relativ simpel und robust sein, da sie nur den lokalen Kontext in Betracht ziehen müssen. Der globale Kontext wird nur in der Integrationsphase wichtig, wenn sich das tentative, inkohärente Netzwerk, das durch die kontext-freien Konstruktionsregeln geformt wurde, in einen stabilen Zustand überführt".
} 
für eine korrekte (Übersetzung und) Interpretation unerlässlich sei. Quine (1960, S. 59) betont zum Beispiel:

"The maxim of translation underlying all this is that assertions startlingly false on the face of them are likely to turn on hidden differences of language. [...] The common sense behind the maxim is that one's interlocutor's silliness, beyond a certain point, is less likely than bad translation - or, in the domestic case, linguistic divergence".

Donald Davidson (1984, S. 27) schlägt das Nachsichtsprinzip, "the principle of charity", vor:

"Charity in interpreting the words and thoughts of others is unavoidable in another direction as well: just as we must maximize agreement, or risk not making sense of what the alien is talking about, so we must maximize self-consistency we attribute to him, on pain of not understanding him."

Grandy (1973, S. 443) schlägt das "principle of humanity" vor: die Forderung, dass das Muster der Relationen von Überzeugungen, Wünschen und der Welt, das dem Autor zugeschrieben wird, dem eigenen Muster so ähnlich wie möglich sein muss.

Keines dieser Prinzipien, die in der Diskussion stehen, ist grundlegend neu. Schon im Jahre 1654 hat Johannes Clauberg in bewundernswerter Ausführlichkeit im Kapitel XIII des dritten Teils seiner Logica, Vetus \& Nova, Prinzipien des "in bonam partem interpretari" herausgearbeitet, das Nachsichtsprinzip - "benignitas" - war dabei das wichtigste. Und bereits 1757 hatte Georg Friedrich Meier (1757/1996, §39) das Prinzip der hermeneutischen Billigkeit als das allgemeinste Interpretationsprinzip aller Interpretationsregeln einer hermeneutica universalis vorgeschlagen:

"Die hermeneutische Billigkeit (aequitas hermeneutica) ist die Neigung eines Auslegers, diejenigen Bedeutungen für hermeneutisch wahr zu halten, welche mit den Vollkommenheiten des Urhebers des Zeichens am besten übereinstimmen, bis das Gegenteil erwiesen ist".

Es ist wichtig zu betonen, dass das Prinzip der hermeneutischen Billigkeit explizit als Präsumtion formuliert ist: als eine Regel, die an der Erfahrung scheitern kann. In der 
angelsächsischen Diskussion über radikale Interpretation, die ich vorher erwähnte, liegt die Betonung darauf, dass die jeweiligen Prinzipien konstitutiv für die Interpretationspraxis sind. Sie werden somit mit einem speziellen Status versehen, der sie für jede Interpretationspraxis unentbehrlich machen sollte bzw. der als allgemeine Voraussetzung anzuerkennen wäre. Ob die Inanspruchnahme dieser Präsumtionsregeln für die Interpretationspraxis konstitutiv ist, sei dahingestellt. Mir scheint jedenfalls, dass der Anschein ihrer Unverzichtbarkeit darauf zurückzuführen ist, dass sie sich besonders gut bewährt haben, weil sie sehr oft mit Erfolg verwendet worden sind (Mantzavinos, 2006, S. 120). Es ist also nur ihr hoher Bewährungsgrad, der dazu veranlasst, anzunehmen, dass sie unverzichtbar für jede Interpretation sind. ${ }^{5}$

Die zweite Argumentationslinie hinsichtlich der Unerlässlichkeit normativer Voraussetzungen für die Interpretationspraxis bezieht sich auf die Unentbehrlichkeit einer Rationalitätsannahme in jeglicher Interpretation (Livingston 1993). Es ist laut diesem Argument nur dann möglich, sprachliche Äußerungen zu erfassen, wenn man unterstellt, dass die Sprecher oder Autoren komplexe Eigenschaften aufweisen, die auf angemessene Art und Weise als rational zu konzeptualisieren sind. Die deduktive Rationalität spielt hier eine wichtige Rolle: es wird unterstellt, dass bei der Produktion sprachlicher Äußerungen, die Schlussregeln der Aussagen- und Prädikatenlogik respektiert werden müssen. Nur in diesem Fall sei die Aneignung des Sinnes von Texten und sprachlichen Äußerungen im Allgemeinen möglich (Føllesdal, 1982, S. 311). Gemäß dieser Auffassung ist die Rationalität konstitutiv für die Überzeugungen des Autors, die zu der Generierung seiner sprachlichen Äußerungen führen und somit macht die Rationalität die normative Voraussetzung aus, die jeder Interpretationspraxis zugrunde liegen muss. Die Rationalitätsannahme (in diesem Kontext) ist jedoch sicherlich kein unangreifbares Prinzip und die Frage, ob Rationalität in der Tat konstitutiv ist, wenn (erfolgreiche) Interpretation stattfinden soll, muss vorerst offen bleiben (Scholz, 2016, S. 228ff.).

\footnotetext{
5 Hirsch (1967, S. 203) betont den heuristischen Charakter der Interpretationsregeln bzw. Maximen. Zur heuristischen Funktion von Interpretationsprinzipien vgl. auch Scholz (2016, S. 163).
} 
Insofern ist festzuhalten, dass der Prozess der Textinterpretation, der im Zentrum der Hermeneutik als methodologische Disziplin der Interpretation liegt, empirisch mit der Hilfe überprüfbarer Modelle analysiert werden kann. Die Frage ob es bestimmte normative Voraussetzungen der Interpretationspraxis gibt - wie spezifische Interpretationsprinzipien, die konstitutiv für diese Praxis sind, oder unerlässliche Rationalitätsprinzipien - ist eine zentrale Angelegenheit von offensichtlicher philosophischer Bedeutung. Unabhängig davon, welche Position man diesbezüglich einnehmen möchte, ist es kaum möglich, zu negieren, dass die Interpretationspraxis viele Formen annehmen und im Hinblick auf diverse Ziele stattfinden kann, ein Problembereich, dem ich mich jetzt zuwenden möchte.

\section{Ziele der Interpretation}

Wir haben gesehen, dass die Textinterpretation über die Interpretation einfacher oder komplexer Sätze hinausgeht, da sie eine Anzahl von Inferenzen beinhaltet, die nötig sind, um sich den Sinn eines Textes anzueignen. Textinterpretation als zielgerichtete Tätigkeit kann unterschiedliche Formen annehmen, aber muss auf jeden Fall von der Textkritik strikt unterschieden werden. Etliche Konfusionen können leicht geklärt werden, wenn man die Interpretation als eine Tätigkeit, die auf die Erfassung des Sinnes eines Textes abzielt, von der Textkritik als einer Tätigkeit, die die Relevanz des Textes hinsichtlich unterschiedlicher Wertgesichtspunkte zum Gegenstand hat, unterscheidet. Hirsch (1967, S. 7f.) hat zu Recht auf dieser Unterscheidung zwischen Sinn und Bedeutsamkeit bestanden:

"Wahrscheinlich die extremsten Beispiele dieses Phänomens sind Fälle von Zurückweisung durch den Autor selbst, wie zum Beispiel Arnolds öffentlicher Angriff auf sein Meisterwerk Empedocles on Etna, oder Schellings Ablehnung all seiner vor 1809 geschriebenen Philosophie. In diesen Fällen kann nicht der geringste Zweifel herrschen, dass des Autors spätere Stellungnahme zu seinem Werke von seiner ursprünglichen stark abwich. Anstatt schön, profund, oder brilliant zu erscheinen, schien 
das Werk fehlgeleitet, trivial und falsch, und sein Sinn war nicht mehr der, den der Autor mitteilen wollte. Alle diese Beispiele zeigen nicht, dass sich der Sinn des Textes verändert hat, ganz im Gegenteil. Wenn sich der Sinn des Textes verändert hätte, dann hätte der Autor nicht seinen Sinn zurückweisen müssen und sich die Unannehmlichkeit eines öffentlichen Widerrufs ersparen können. Zweifellos hatte sich die Relevanz der Arbeit für den Autor enorm verändert, aber sein Sinn überhaupt nicht."

Auch wenn man den Unterschied zwischen Sinn und Relevanz (oder Bedeutsamkeit) anerkennen möchte, und die Entscheidung trifft, die Unterscheidung zwischen Textinterpretation und Textkritik zu respektieren, ist es unumstritten, dass die Interpretation im Hinblick auf viele unterschiedliche Ziele ausgerichtet werden kann. Für lange Zeit hat sich die Diskussion über das angemessene Ziel der Interpretation um den sogenannten "intentionalistischen Fehlschluß" gedreht, vor allem in der klassischen Formulierung von Wimsatt and Beardsley (1946, S. 468): "[T]he design or intention of the author is neither available nor desirable as a standard for judging the success of a work of literary art". ${ }^{6}$ Der Kern der Debatte bezieht sich auf die Frage, ob die Erfassung der Autorintention eines Textes das einzige Interpretationsziel ist oder nicht und, vorausgesetzt, dass die Autorintention in der Tat das Ziel der Interpretation ist, wie genau sie erfasst werden kann. Die wesentliche Frage mit der wir konfrontiert werden, wenn wir irgendeinen Text studieren, ist, wie Quentin Skinner einflussreich argumentiert hat, die folgende (1969: 48f.):

"what its author, in writing at the time he did write for the audience he intended to address, could in practice have been intending to communicate by the utterance of this given utterance. It follows that the essential aim, in any attempt to understand the utterances themselves, must be to recover this complex intention on the part of the author. And it follows from this that the appropriate methodology for the history of ideas must be concerned, first of all, to delineate the whole range of communications which could have been conventionally performed on the given occasion by the utterance of the given

${ }^{6}$ In späteren Veröffentlichungen hat Wimsatt den "intentionalistischen Fehlschluß" anders formuliert. Für eine Diskussion vgl. Hermerén (1974, S. 60ff.). 
utterance, and, next, to trace the relations between the given utterance and this wider linguistic context as a means of decoding the actual intention of the given writer."

Außer Quentin Skinner (1972, 1975), haben auch Axel Bühler und andere die These vertreten, die ich auch teile, nämlich, dass die Intention des Autors ermittelbar ist. Es ist grundsätzlich möglich, Autorabsichten festzustellen, solange die Quellenlage und die Textüberlieferung es erlauben (Bühler, 1999a, S. 62ff.). Sogar bei fiktionaler Rede kann man die (kommunikative) Absicht des Autors dahingehend spezifizieren, dass er seine Adressaten zu einer Einstellung des So-tun-als-ob gegenüber den Inhalten der fiktionalen Rede bewegen will. Zudem hat die Kontroverse zum "Hermeneutischen Intentionalismus" gezeigt, dass die Ermittlung der Intention des Autors ein durchaus verfolgenswertes Ziel der Interpretation darstellt. Insofern ist der intentionalistische Fehlschluß kein echter Fehlschluss, weil die Ermittlung der Intention des Autors ein sowohl mögliches als auch wünschenswertes Interpretationsziel darstellt.

Obwohl der Begriff der Intention bestimmt nützlich für eine methodologische Konzeption von Interpretation ist, ist er sicherlich Teil einer späteren Entwicklung; er ist in die hermeneutische Methodologie von Diskussionen in der Sprachphilosophie und der Philosophie des Geistes in der analytischen Tradition des 20. Jahrhunderts importiert worden. Er stellte eine Reaktion gegen zwei Orthodoxien dar, die zu jener Zeit herrschten. Auf der einen Seite, dass die Interpretation nur auf den konkreten Text selbst abzielen sollte; und auf der anderen, dass die Interpretation auf den sozialen Kontext abzielen sollte, der das Zustandekommen des konkreten Textes verursacht hat (Skinner 1969).

Der Begriff des Sinnzusammenhangs, der von Dilthey und anderen Autoren in der klassischen hermeneutischen Tradition gebraucht worden ist, scheint mir jedoch als terminus technicus geeigneter als der Begriff der Intention. Ein Sinnzusammenhang, der in Verbindung mit einer spezifischen sprachlichen Äußerung oder einem spezifischen Text steht, wird vom Autor vor dem Hintergrund seiner Ziele, seiner Überzeugungen und seiner übrigen mentalen Zustände in Wechselwirkung mit seiner natürlichen und sozialen Umgebung konstruiert: diese Sinnkonstruktion ist ein komplexer Vorgang und umfasst 
die bewusste oder unbewusste Verwendung von Symbolen. Die Textinterpretation kann als diejenige Aktivität konzeptualisiert werden, die auf die korrekte Identifikation des Textsinnes abzielt, vermöge der zutreffenden Rekonstruktion des Sinnzusammenhanges, der in Verbindung mit dem Text entstanden ist. Die Möglichkeit der Beschreibung des Sinnzusammenhanges mittels des Begriffes der Intention ist eine legitime, aber sicherlich nicht die einzige Möglichkeit. Es kann durchaus der Fall sein, dass die Spezifizierung der Intention des Autors für die Beschreibung des Sinnzusammenhangs adäquat ist, aber die Rekonstruktion des Sinnzusammenhangs kann komplexer sein. Es ist, mit anderen Worten, nicht nötig, sich bei der Rekonstruktion eines Sinnzusammenhanges eines bestimmten Beschreibungssystems zu bedienen: der Prozess der Rekonstruktion braucht nicht an den Begriff der Intention gebunden zu sein. Da das, was rekonstruiert werden muss, ein Sinnzusammenhang ist, kann ein komplett unterschiedlicher Begriffsapparat gebraucht werden. Es ist möglich, die Intention des Autors nebst einer Analyse grammatikalischer und anderer Elemente $\mathrm{zu}$ verwenden, um eine adäquate Rekonstruktion zu erreichen.

Der Begriff des Sinnzusammenhanges ist zentral für die hermeneutische Methodologie, vor allem deshalb, weil er auf die hermeneutische Praxis einer Reihe von Disziplinen anwendbar ist. Coseriu (1994/2006) hat in seiner einflussreichen Textlinguistik den Begriff des Umfeldes verwendet, um dasselbe zu bezeichnen, dass ich mit dem Begriff des Sinnzusammenhangs bezeichnet habe. Die Rekonstruktion des "Umfeldes" - in der Tradition des klassischen Organon Models von Karl Bühler, der von "sympraktischem, symphysischem und synsemantischem Umfeld" (1934/1965, S. 154ff.) sprach - zielt auf die Erfassung des Textsinnes mittels der Beschreibung des ganzen Kontextes, soweit es möglich ist. Es ist somit offensichtlich, dass die Interpretation in der hermeneutischen Tradition als ein Prozess der Rekonstruktion eines Sinnzusammenhangs konzeptualisiert wird und somit das Gegenteil des Prozesses der Dekonstruktion darstellt, wie er von Derrida und seinen Anhängern vorgeschlagen wird. Wie Rescher zutreffend diesbezüglich darstellt (1997, S. 201):

"Der Hauptpunkt ist also, dass jeder Text in einem historischen und kulturellen Kontext steht, und dass der Kontext eines Textes nicht selbst textlich ist - nicht etwas ist, dass 
alleine und komplett im textlichen Bereich ausgetragen werden kann. Dieser Kontext der Texte, der uns interessiert, bedingt und schränkt die plausiblen Interpretationen ein, die mit diesen Texten kompatibel sind. Der Prozess der Dekonstruktion - des interpretativen Auflösens eines jeden Textes in eine Pluralität von gleich verdienstvollen Konstruktionen - kann und sollte durch den Prozess der Rekonstruktion aufgehoben werden, die fordert, Texte in deren größeren Kontext zu betrachten. Schließlich haben Texte unvermeidlich eine Kulisse - historische, kulturelle, des Autors - von denen ihr tatsächlicher Sinn kritisch abhängt.“ [Meine Übersetzung]"

Die Auffassung von Interpretation als Prozess der Rekonstruktion des Sinnzusammenhangs eines Textes schenkt dem Kontext des Textes die gebührende Aufmerksamkeit, ohne jedoch anzunehmen, dass der soziale und historische Kontext die Produktion des Textes verursacht haben. Diese Auffassung erlaubt auch die Versöhnung bzgl. einer anderen Facette der alten Kontroverse hinsichtlich der Ziele der Interpretation. Ich habe vorher erwähnt, dass die Frage, ob die Erfassung der Autorintention das einzige legitime Interpretationsziel ausmacht, für lange Zeit der Gegenstand einer heftigen Kontroverse war $^{7}$. Diese Kontroverse scheint mir durchaus lösbar zu sein, sobald man sich den technologischen Charakter der Hermeneutik vor Augen führt (Albert, 2003).

\footnotetext{
${ }^{7}$ Eine Kontroverse über dieses Thema wurde zum Beispiel in einigen Ausgaben der Zeitschrift Poetics Today ausgetragen, die der Fruchtbarkeit der Kognitionswissenschaften für die Literaturtheorie gewidmet waren. Einige Parteien waren dagegen, die Erkenntnisse der Kognitionswissenschaft in der Literaturwissenschaft anzuwenden, einige Parteien waren dafür, aber die meisten Parteien schienen diesbezüglich verwirrt zu sein. Es ist m.E. aus den beiden folgenden Zitaten ersichtlich, dass die Autoren es, unter anderem, ablehnen, sich auf spezifische Ziele von (literarischen) Interpretationen zu verpflichten. Adler und Gross, in ihrer Kritik des Kognitivismus in der Literaturwissenschaft, führen aus (2002, S. 214): "Es ist anzumerken, dass die Kategorie der Wahrheit nicht in der Gleichung auftaucht; hier geht es um die Adäquatheit des Ansatzes, und diese wiederum hängt von persönlichen Ansichten über das Thema ab. Literaturanalyse ist viel weniger auf die Korrektheit oder die Wahrscheinlichkeit von Erkenntnissen oder die Unbestreitbarkeit der Evidenz ausgerichtet. Vielmehr besteht ihr 'Erfolg' in solchen Parametern wie Originalität, Angemessenheit, Einfallsreichtum, oder 'Einsichts-wert': dies kann bemessen werden anhand unseres Grades der Zufriedenheit damit, was über den Text herausgefunden wird oder wie er beleuchtet wird." Die Herausgeber des ursprünglichen Bandes, Alan Richardson and Francis Steen, bemerken in ihrer Replik auf Adler und Gross (2003, S. 155): „Es ist unsere feste Überzeugung, dass die Wissenschaft keine autoritativen Antworten über den Sinn und die Relevanz von literarischen Werken liefern kann und soll. [...] Sich wesentlich für die Modelle, Theorien und Erkenntnisse zu interessieren, die aus Arbeiten in der Kognitionswissenschaft und den Neurowissenschaften stammen, verpflichtet einen nicht zu einer
} 
Ihr technologischer Charakter manifestiert sich auf positive Weise, indem man die Pluralität der Interpretationsziele positiv anerkennt. Die für die Tätigkeit der Interpretation in Betracht kommenden Zielsetzungen müssen nicht auf einen einzigen Nenner gebracht werden und es müssen auch nicht einige zugunsten von anderen geopfert werden. Obwohl natürlich eine Debatte über die Bedeutsamkeit der unterschiedlichen Interpretationsziele durchaus mit rationalen Argumenten geführt werden kann, braucht diese nicht zu endgültigen Resultaten zu führen, die für alle verbindlich sein sollten. Dies wird kaum je der Fall sein, da ein Konsens über die geeigneten Interpretationsziele typischerweise provisorischer Natur sein wird: es genügt vielmehr, bestimmte Ziele der Interpretation als hypothetisch gültig anzunehmen und dann die alternativen Hypothesen im Hinblick auf das jeweilige Ziel zu formulieren und zu testen. Man braucht, mit anderen Worten, ein bestimmtes Ziel nur als hypothetisch gültig anzunehmen, um dann herauszufinden, wie dies erreicht werden kann. Solch eine Technologie operiert mit hypothetischen und nicht mit kategorischen Imperativen. Die Maßstäbe für die komparative Bewertung der Interpretationshypothesen können jeweils an unterschiedlichen regulativen Ideen orientiert sein. Man kann zum Beispiel die Rekonstruktion des Sinnzusammenhangs eines Textes im Hinblick auf die Idee der Wahrheit vornehmen: Interpretationsaktivitäten würden dann auf die zutreffende Darstellung des Sinnzusammenhangs des Textes abzielen. Aber man kann einen Text auch hinsichtlich anderer Ziele interpretieren, zum Beispiel Ziele ästhetischer Art und in diesem Fall wären andere Maßstäbe und regulative Ideen maßgeblich, etwa die der Schönheit ${ }^{8}$. Ob Wahrheit oder Schönheit das legitime Ideal für die Beurteilung einer Interpretation sein soll, ist ein Diskurs, der sich auf einer anderen Ebene entfalten kann und der nicht mittels einer dogmatischen Dezision ein für alle Mal entschieden zu werden

wissenschaftlichen Methodologie, genauso wenig, wie ein Interesse an der Psychoanalyse Freudianische oder Lacansche Literaturkritiker zu einer therapeutischen Disziplin verpflichtet.“ [Meine Übersetzung]

${ }^{8}$ In der Literatur ist die Behauptung verbreitet, dass Texte hinsichtlich anderer Ziele als der Wahrheit interpretiert werden sollen, eine Behauptung, die sowohl plausibel als auch korrekt ist. Aber in der Hermeneutik als technologischer Disziplin kann eine gewisse Menge an Interpretationen mit Bezug auf verschiedene Standards oder Kriterien beurteilt werden, und einige erfüllen diese, andere nicht. Eine Interpretation kann zum Beispiel originell und gleichzeitig falsch sein.“ [Meine Übersetzung] 
braucht. Im Gegensatz zur "Autorintention" stellt der "Sinnzusammenhang" ein komplexes Phänomen dar und die Interpreten können sich entscheiden, ihn hinsichtlich unterschiedlicher Ziele und Maßstäbe zu beleuchten und zu erfassen - und das ist auch

oft der Fall. Was den Kern dieser epistemischen Aktivität ausmacht, d.h. die Erfindung und Überprüfung von Interpretationshypothesen zur Rekonstruktion von Sinnzusammenhängen im Hinblick auf unterschiedliche Ziele, sowie das entsprechende methodische Vorgehen, ist was uns im letzten Teil dieses Aufsatzes beschäftigen wird.

\section{Die hypothetisch-deduktive Methode}

Die Hermeneutik als generelle Methode der Interpretation von menschlichen Handlungen und den Produkten dieser Handlungen kann sich der hypothetisch-deduktiven Methode bedienen. Meine These lautet, dass diese Methode genauso fruchtbar als Methode der Interpretation zu verwenden ist, wie sie auch als naturwissenschaftliche Methode verwandt wurde. Die hermeneutische Methode ist die hypothetisch-deduktive Methode in ihrer Anwendung auf bedeutungstragende Entitäten und Phänomene (meaningful Material) (Føllesdal, 1979). Das Grundcharakteristikum dieser Methode ist darin zu sehen, dass wissenschaftliches Arbeiten im Allgemeinen in einem Bezug zu Hypothesen steht. Möglicherweise können Hypothesen Phänomene erklären, die hypothetischdeduktive Methode ist aber nicht mit einem Modell wissenschaftlicher Erklärung identisch (Føllesdal, Walløe, und Elster, 1996, S. 93ff.). Hempel und Popper (1935/2005; 1963/1989), welche die Protagonisten der akribischen Bearbeitung dieser Methode für die moderne Wissenschaftstheorie waren, haben zum großen Teil wissenschaftliche Aktivität mit der Bereitstellung von Erklärungen gleichgesetzt. Es besteht aber kein Grund anzunehmen, dass Feststellungen von Tatbeständen von geringerem wissenschaftlichen Interesse sind.

Meine These lautet, dass wissenschaftliche Problembearbeitung nicht nur dadurch charakterisiert ist, dass man Antworten auf "Warum?" - Fragen sucht, sondern auch dadurch, dass man auf Fragen der Art: "Was ist der Fall?" bzw. "Was war der Fall?" eine 
Antwort sucht. Es geht darum, einzelne Sachverhalte bzw. Einzeltatsachen in zutreffender Weise festzustellen, im hier interessierenden Fall also einen Sinnzusammenhang, der mit einer bestimmten sprachlichen Äußerung bzw. einem bestimmten Text verknüpft ist. Die Anwendung der hypothetisch-deduktiven Methode ist ein Weg nachzuweisen, dass dieselben Maßstäbe, die momentan für die Behandlung von Erklärungsproblemen verwendet werden - intersubjektive Verständlichkeit, Überprüfbarkeit mithilfe von Evidenz, Austausch von Argumenten und Objektivität auch auf Interpretationsprobleme anwendbar sind. Insofern man bereit ist, in der Anwendung solcher Maßstäbe die Wissenschaft als rationales Unternehmen anzuerkennen, wäre auch die Interpretation als ein rationales Unternehmen anzuerkennen und die Hermeneutik als rationale Methodenlehre der Interpretation zu bezeichnen. Ich werde sehr kurz skizzieren, wie diese Methode in fünf Schritten angewandt werden kann.

Eine Rekonstruktion stellt einen Beschreibungsversuch dar, also den Versuch einer zutreffenden Darstellung singulärer Ereignisse, und ist eine Antwort auf eine Frage der Art "Was ist der Fall?". Wie bereits betont, behaupten Antworten auf Fragen dieser Art das Vorliegen von einzelnen Sachverhalten bzw. von Einzeltatsachen. Sie sind singuläre deskriptive Sätze, die einzelne Ereignisse betreffen und raum-zeitlich bestimmt sind. Die Rekonstruktion des Sinnes eines Textes stellt somit einen Beschreibungsversuch dar, der darauf abzielt, den Sinnzusammenhang dieses bestimmten Textes zutreffend darzustellen. Da einem Text dadurch Sinn verliehen worden ist, dass der Autor ihn vor dem Hintergrund seiner Ziele, seiner Überzeugungen und seiner übrigen mentalen Zustände mittels Symbolverwendung gedeutet hat, geht es darum, diesen Sinn festzustellen, indem man die grundlegenden Elemente dieses Zusammenhangs zutreffend rekonstruiert. Um dies zu erreichen stellt man Intepretationshypothesen auf, und dies ist der erste Schritt der Anwendung der hypothetisch-deduktiven Methode.

Das Aussagesystem, das diese Interpretationshypothesen konstituiert, besitzt einen prinzipiell hypothetischen Charakter, weil es nicht sicher ist, ob es im Hinblick auf die Erreichung des Erkenntniszieles, nämlich der Feststellung des Sinnes des Textes, zutreffend ist oder nicht. Die Produktion von Deutungshypothesen, die den Sinn des Textes eruieren sollen, ist, entgegen der vorherrschenden Meinung, kein Verfahren, dem 
bestimmte Algorithmen zugrunde liegen. In der Konstruktion solcher Hypothesen kann man von diversen Interpretationsprinzipien, wie z.B. denjenigen, die wir bereits diskutiert haben - dem Nachsichtprinzip, dem "Principle of Humanity" usw. - Gebrauch machen. Aber all diese besitzen den Charakter von Präsumtionen, die an der Erfahrung scheitern können. Auf jeden Fall können solche Interpretationshypothesen zum Teil aus nicht beobachtbaren "theoretischen Termen" (theoretical terms) bestehen, welche sich, zum Beispiel, auf die Intention des Autors beziehen können. In solchen Fällen erfolgt, in einem zweiten Schritt, eine Ableitung von Konsequenzen aus diesen Interpretationshypothesen (mit Hilfe weiterer Annahmen, Theorien, Gesetze usw.). Diese Konsequenzen können leichter überprüfbar sein.

Im dritten Schritt werden diese beobachtbaren Konsequenzen mit Hilfe des zur Verfügung stehenden empirischen Materials geprüft, zu dem auch Belege über einzelne Handlungen und Äußerungen gehören können. Diese Evidenz wird aufgrund der Anwendung der diversen Forschungstechniken der Sozial- und Geisteswissenschaften bereitgestellt. (Es sollte klar sein, dass die Entwicklung solcher Forschungstechniken natürlich auf nomologischem Wissen basiert. Insofern wird bei der Überprüfung von Interpretationshypothesen, wenn nicht immer explizit, so zumindest implizit nomologisches Wissen herangezogen. Entscheidend ist nur, dass dieses Wissen bzw. die entsprechenden Gesetzesaussagen in anderen als dem vorliegenden Kontext, also unabhängig, überprüft worden sind und sich bewährt haben. Diese Gesetzesaussagen können trivialer Art sein, wie zum Beispiel, dass jedes schriftliche Dokument von Personen geschrieben worden ist, aber das soll nicht heißen, dass sie keine Rolle spielen). Diese Evidenz kann sich auf das, was der Autor selbst über sein Werk gesagt hat, beziehen, aber auch alles Mögliche andere umfassen, was vom Autor je geschrieben worden ist. Außerdem können Details in Reim, Rhythmus oder in anderen literarischen Stilmitteln berücksichtigt werden bzw. die Häufigkeit von Wörtern, die in einem Text vorkommen und ähnliches (Gomperz, 1939, S. 59ff., Føllesdal 1979, S. 324f., Nehamas, 1981, S. 145, Rescher, 1997, S. 200f.). Wichtig ist jedenfalls zu betonen, dass es bei der Aufzählung der unterschiedlichen Merkmale, die die jeweiligen Interpretationshypothesen unterstützen sollen, nicht nur auf die Anzahl dieser Merkmale 
ankommt, sondern auch darauf, um was für Merkmale es sich handelt (Føllesdal, Walløe, Lars und Elster, 1988, S. 114).

In einem vierten Schritt werden die Interpretationshypothesen aufgrund solchen empirischen Materials überprüft. Eine komparative Bewertung wird hier nötig, um "gute" von "schlechten" Interpretationen unterscheiden zu können. Solch eine Bewertung kann im Hinblick auf unterschiedliche Werte stattfinden, sodass die Rekonstruktion eines Sinnzusammenhangs an unterschiedlichen regulativen Ideen orientiert werden kann. Eine solche Idee kann die Wahrheit sein, die als die zutreffende Darstellung eines Sinnzusammenhangs weiter spezifiziert werden kann - die Interpretationen stellen Hypothesen dar, gerade vermöge der Tatsache, dass man Gründe für ihre Wahrheit bzw. Falschheit sucht. ${ }^{9}$ Andere Werte, wie zum Beispiel ästhetische, können auch als wichtig betrachtet werden und die komparative Evaluation der angebotenen Interpretationen kann auch im Hinblick auf solche Werte stattfinden - zum Beispiel Schönheit.

Im fünften Schritt der Anwendung der hypothetisch-deduktiven Methode kann eine multidimensionale Evaluation derselben Interpretationshypothese im Hinblick auf diverse Werte oder diverser Interpretationshypothesen im Hinblick auf denselben Wert stattfinden. Solch eine Evaluation erfolgt nicht mit Hilfe irgendwelcher algorithmischen Verfahren. Die Anwendung spezifischer Kalküle, die angeblich zu determinierten Evaluationen und Entscheidungen führen soll, ist weder im Fall der Textinterpretation noch im Fall der wissenschaftlichen Erklärung möglich. Menschliche Entscheidungen, die Phantasie involvieren, sind bei einer kognitiven Praxis dieser Art am Werke, Entscheidungen, die fehlbar sind. Es ist nur die Institutionalisierung der Möglichkeit von Kritik, die zur Fehlerkorrektur führen kann, solange diese Evaluationen und Entscheidungen involviert sind. Unsere falliblen Urteile sind alles was wir haben, hier und anderswo, und die Ermöglichung einer kritischen Diskussion ist die Voraussetzung, um informierte Entscheidungen treffen zu können.

9 Die Bereitstellung hinreichender empirischer Evidenz ist conditio sine qua non, um Wahrheitsansprüche geltend zu machen. Vgl. die spannende und überzeugende Kritik von Tallis (1999), welche zeigt, dass eine Reihe postmoderner Theoretiker hingegen "evidenzfreie Verallgemeinerungen" von oft kolossalem Umfang zu favorisieren pflegen. 
Es ist wichtig $\mathrm{zu}$ betonen, dass der fünfte Anwendungsschritt dieser Methode eine wichtige Konsequenz hinsichtlich der Blockade eines potentiell wichtigen Problems hat, was erschöpfend in der Bestätigungstheorie behandelt worden ist. Wenn die kontingente Evidenz $E$ die Hypothese $H$ gegeben der Hintergrundannahmen $B$ bestätigt, dann bestätigt $E$ auch die Konjunktion von $H^{\wedge} X$ für jede willkürliche Aussage $\mathrm{X}$, die konsistent mit $\mathrm{H}$ ist. Diese Besonderheit kann den Bestätigungsprozess extrem freizügig machen und somit die ganze Methode unbrauchbar werden lassen. Eine substanzielle kritische Diskussion, die mit Argumenten von unterschiedlichen Textinterpreten ausgeführt wird, ist somit eine conditio sine qua non für die Fruchtbarkeit der hypothetisch-deduktiven Methode. Scholz (2015) hat genau aus diesem Grund die Produktivität dieser Methode hinterfragt - er nennt es "das Relevanzproblem" - und hat vorgeschlagen, dass es mit einem Schluss auf die beste Erklärung gelöst werden kann (Lipton, 2004). Gemäß diesem Vorschlag muss die Alternative, welche am besten die Evidenz erklärt, unter allen vorgeschlagenen Alternativen akzeptiert werden. Die Erfolgschancen dieses Zuges scheinen jedoch fraglich zu sein. Er beruht nämlich auf der Annahme, dass es möglich ist, notwendige und hinreichende Bedingungen für das was eine Erklärung ist, anzugeben und auf der weiteren Annahme, dass es einen universellen Konsens gibt, bezüglich dessen was als "beste Erklärung" gelten soll. Beide Annahmen sind jedoch unhaltbar (Mantzavinos, 2013, 2016).

\section{Epilog}

Abschließend ist festzuhalten, dass die hypothetisch-deduktive Methode weitgehend zur Etablierung hermeneutischer Objektivität führen kann, welche letztlich auf der kritischen Diskussion zwischen den Teilnehmern bzgl. der Angemessenheit der unterschiedlichen Interpretationen im Hinblick auf die Erreichung diverser Interpretationsziele basiert. Intersubjektive Verständlichkeit, Überprüfbarkeit mithilfe von Evidenz, Austausch von Argumenten und Objektivität sind somit möglich im Falle der Textinterpretation, wie eine Reihe von Beispielen aus diversen Disziplinen belegen (Føllesdal, 1979, 
Mantzavinos, 2006, Kap. 6, Detel, 2011, p. 394ff, Detel, 2016). Hermeneutik als rationale Methodenlehre der Interpretation ist somit möglich und fruchtbar. 


\section{Bibliographie}

Adler, Hans und Sabine, Gross (2002): “Adjusting the Frame: Comments on Cognitivism and Literature", in: Poetics Today, Bd. 23:2, S. 195-220

Albert, Hans (2003): "Hermeneutik und Realwissenschaft. Die Sinnproblematik und die Frage der theoretischen Erkenntnis", in: (ed.) Axel Bühler: Hermeneutik. Basistexte zur Einführung in die wissenschaftstheoretischen Grundlagen von Verstehen und Interpretation", Heidelberg: Synchron, pp. 23-58.

Anderson, Lanier (2003): "The Debate over the Geisteswissenschaften in German Philosophy, 1880-1910" in: Baldwin, Thomas (ed.): The Cambridge History of Philosophy: 1870-1945, Cambridge: Cambridge University Press, pp. 221-234.

Bühler, Axel (1993): "Der Hermeneutische Intentionalismus als Konzeption von den Zielen der Interpretation", in: Ethik und Sozialwissenschaften, vol. 4, pp. 511-518.

Bühler, Axel (1999a): "Autorabsicht und fiktionale Rede", in: (eds.) Jannidis, Fotis, Gerhard Lauer, Matias Martinez, and Simone Winko: Rückkehr des Autors. Zur Erneuerung eines umstrittenen Begriffs, Tübingen: Max Niemeyer Verlag, pp. 61-75.

Bühler, Axel (1999b): "Die Vielfalt des Interpretierens", in: Analyse und Kritik, vol. 21, pp. 117-137.

Bühler, Axel (2003): "Grundprobleme der Hermeneutik", in: (ed.) Axel Bühler: Hermeneutik. Basistexte zur Einführung in die wissenschaftstheoretischen Grundlagen von Verstehen und Interpretation, Heidelberg: Synchron, pp. 3-19.

Bühler, Karl (1934/1965): Sprachtheorie. Die Darstellungsfunktion der Sprache, 2. Auflage, Stuttgart: Gustav Fischer Verlag.

Clauberg, Johannes (1654): Logica, Vetus \& Nova, Amsterdam: Ex Officin Elzeviriana.

Coseriu, Eugenio (2006): Textlinguistik, 4. Auflage, Tübingen: Francke Attempto.

Davidson, Donald (1984): Inquiries into Truth \& Interpretation, Oxford: Oxford University Press.

Detel, Wolfgang (2011): Geist und Verstehen, Frankfurt am Main: Vittorio Klostermann.

Detel, Wolfgang (2014): Kognition, Parsen und rationale Erklärung: Elemente einer allgemeinen Hermeneutik, Frankfurt am Main: Vittorio Klostermann. 
Detel, Wolfgang (2016): Hermeneutik der Literatur und Theorie des Geistes. Exemplarische Interpretationen poetischer Texte, Frankfurt am Main: Vittorio Klostermann.

Dilthey, Wilhelm (1883/1990): Gesammelte Schriften, I. Band: Einleitung in die Geisteswissenschaften, 9. Auflage, Stuttgart: B.G. Teubner Verlagsgesellschaft und Göttingen: Vandenhoeck \& Ruprecht.

Dilthey, Wilhelm (1924/1990): Gesammelte Schriften V. Band: Die geistige Welt. Einleitung in die Philosophie des Lebens. Erste Hälfte: Abhandlungen zur Grundlegung der Geisteswissenschaften, 8. Auflage, Stuttgart: B.G. Teubner Verlagsgesellschaft und Göttingen: Vandenhoeck \& Ruprecht.

Dilthey, Wilhelm (1927/1992): Gesammelte Schriften VII. Band: Der Aufbau der geschichtlichen Welt in den Geisteswissenschaften, 8. unv. Auflage, Stuttgart: B.G. Teubner Verlagsgesellschaft und Göttingen: Vandenhoeck \& Ruprecht.

Føllesdal, Dagfinn (1979): "Hermeneutics and the hypothetico-deductive method", in: Dialectica, vol. 33, pp. 319-336.

Føllesdal, Dagfinn (1982): "The Status of Rationality Assumption in Interpretation and in the Explanation of Action", in: Dialectica, vol. 36, pp. 301-316.

Føllesdal, Dagfinn, Walløe, Lars and Elster, John (1988): Rationale Argumentation. Ein Grundkurs in Argumentations - und Wissenschaftstheorie, Berlin und New York: Walter de Gruyter.

Føllesdal, Dagfinn, Walløe, Lars and Elster, John (1996): Argumentasjonsteori, språk og vitenskapsfilosofi, Oslo: Universitetsforlaget.

Frege, Gottlob (1884): Die Grundlagen der Arithmetik, Breslau: Verlag von Wilhelm Koebner.

Gadamer, Hans-Georg (1960/1990): Gesammelte Werke, Bd. 1, Hermeneutik I: Wahrheit und Methode, 6. Auflage, Tübingen: J.C.B. Mohr (Paul Siebeck).

Gadamer, Hans-Georg (1986/1993): Gesammelte Werke, Bd. 2, Hermeneutik II: Wahrheit und Methode, Ergänzungen, Register, 2. Auflage, Tübingen, J.C.B. Mohr (Paul Siebeck).

Gadamer, Hans-Georg (2000): Hermeneutische Entwürfe, Tübingen: J.C.B. Mohr (Paul Siebeck).

Gomperz, Heinrich (1939): Interpretation. Logical analysis of a method of historical research, Chicago: The University of Chicago Press. 
Grandy, Richard (1973): "Reference, Meaning, and Belief", in: Journal of Philosophy, vol. 70, pp. 439-452.

Heidegger, Martin (1923/1995): Ontologie (Hermeneutik der Faktizität), Gesammtausgabe, Band 63, 2. Auflage, Frankfurt am Main: Vittorio Klostermann.

Heidegger, Martin (1927/1993): Sein und Zeit, 17. Auflage, Tübingen: Niemeyer.

Hermerén, Göran (1974): "Intention und Interpretation in Literary Criticism", in: New Literary History, Bd. 8, S. 57-82.

Hirsch, E.D. Jr. (1967): Validity in Interpretation, New Haven and London: Yale University Press.

Kintsch, Walter (1998): Comprehension: A Paradigm for Cognition, Cambridge: Cambridge University Press.

Kintsch, Walter and Teun A. van Dijk (1978): "Toward a Model of Text Comprehension and Production", in: Psychological Review, vol. 85, pp. 363-394.

Lewis, David (1983): "Radical Interpretation", in: (ed.) Lewis, David: Philosophical Papers Vol. I, Oxford: Oxford University Press, pp. 108-121.

Lipton, Peter (2004): Inference to the Best Explanation, 2nd edition, London and New York.

Livingston, Paisley (1993): "Why Realism Matters: Literary Knowledge and the Philosophy of Science", in: George Levine (ed.): Realism and Representation: Essays on the Problem of Realism in Relation to Science, Literature, and Culture, Madison: University of Wisconsin Press, pp. 134-154.

Malpas, Jeff and Gander, Hans-Helmuth (eds.) (2014): The Routledge Companion to Hermeneutics, London and New York: Routledge.

Mantzavinos, C. (2006): Naturalistische Hermeneutik, Tübingen: Mohr Siebeck.

Mantzavinos, C. (2012): "Explanations of Meaningful Actions", in: Philosophy of the Social Sciences, vol. 42, pp. 224-238.

Mantzavinos, C. (2013): "Explanatory Games", in: Journal of Philosophy, vol. CX, pp. 606-632.

Mantzavinos, C. (2014): "Text Interpretation as a Scientific Activity", in: Journal for General Philosophy of Science, vol. 45, pp. 45-58. 
Mantzavinos, C. (2016): Explanatory Pluralism, Cambridge: Cambridge University Press.

Meier, Georg Friedrich (1757/1996): Versuch einer allgemeinen Auslegungskunst, mit einer Einleitung und Anmerkungen, Axel Bühler und Luigi Cataldi Madonna (Hrsg.): Hamburg: Felix Meiner Verlag.

Mill, John Stuart (1843/1974): A System of Logic Ratiocinative and Inductive, The Collected Works of John Stuart Mill, vol. VIII, Toronto: University of Toronto Press.

Nehamas, Alexander (1981): "The Postulated Author: Critical Monism as a Regulative Ideal", in: Critical Inquiry, vol. 8, pp. 133-149.

Popper, Karl R. (1935/2005): Logik der Forschung, 11. Auflage, Tübingen: Mohr Siebeck.

Popper, Karl R. (1963/2009): Vermutungen und Widerlegungen, 2. Auflage, Tübingen: Mohr Siebeck.

Quine, Willard van Orman (1960): Word and Object, Cambridge/MA: The MIT Press.

Rescher, Nicholas (1997): Objectivity. The Obligations of Impersonal Reason, Notre Dame, IN and London: University of Notre Dame Press.

Ricoeur, Paul (1981): "What is a Text? Explanation and Intepretation", in: Paul Ricoeur: Hermeneutics and the Human Sciences, ed. and transl. by John B. Thompson, Cambridge: Cambridge University Press, pp.135-151.

Rickert, Heinrich (1929): Die Grenzen der naturwissenschaftlichen Begriffsbildung. Eine logische Einleitung in die historischen Wissenschaften, 5. Auflage, Tübingen: J.C.B. Mohr (Paul Siebeck).

Scholz, Oliver R. (2012): "On the Very Idea of a Textual Meaning", in: (eds.) Daiber, Jürgen, Konrad, Eva-Maria, Petraschka, Thomas and Rott, Hans: Understanding Fiction: Knowledge and Meaning in Literature, Münster: Mentis, pp. 135-145.

Scholz, Oliver R. (2015): "Texte interpretieren - Daten, Hypothesen und Methoden", in: Borkowski, Jan, Descher, Jan, Ferder, Felicitas, Heine, Philipp David (eds.): Literatur intepretieren: Interdisiplinäre Beiträge zur Theorie und Praxis, Münster: Mentis, pp. 147-171.

Scholz, Oliver R. (2016): Verstehen und Rationalität, 3. rev. Auflage, Frankfurt am Main: Vittorio Klostermann. 
Skinner, Quentin (1969): "Meaning and Understanding in the History of Ideas", in: History and Theory, vol. 8, pp. 3-53.

Skinner, Quentin (1972): "Motives, Intentions and the Interpretation of Texts", in: New Literary History, vol. 3, pp. 393-408.

Skinner, Quentin (1975): "Hermeneutics and the Role of History", in: New Literary History, vol. 7, pp. 209-232.

Szabó, Zoltán Gendler (2013): "Compositionality", The Stanford Encyclopedia of Philosophy, Edward N. Zalta (Hrsg.).

Tallis, Raymond (1999): "Evidence-Based and Evidence-Free Generalizations: A Tale of two Cultures", in: (Hrsg.) Fuller, David und Patricia Waugh: The Arts and Sciences of Criticism, Oxford and New York: Oxford University Press, S. 71-93.

Weber, Max (1922/1985): Gesammelte Aufsätze zur Wissenschaftslehre, 6th. rev. edition, J. C .B. Mohr (Paul Siebeck).

Wimsatt, William Jr. and Monroe C. Beardsley (1946): "The Intentional Fallacy", in: Sewanee Review, vol. 54, pp. 468-488.

Windelband, Wilhelm (1894/1915): "Geschichte und Naturwissenschaft", in: Windelband, Wilhelm (ed.): Präludien. Aufsätze und Reden zur Philosophie und ihrer Geschichte, Band 2, 5. erweiterte Auflage, Tübingen: J.C.M. Mohr (Paul Siebeck), pp. 136-160. 